\title{
CORRECTION
}

\section{Correction: Technical standards for the interpretation and reporting of constitutional copy-number variants: a joint consensus recommendation of the American College of Medical Genetics and Genomics (ACMG) and the Clinical Genome Resource (ClinGen)}

Erin Rooney Riggs, Erica F. Andersen, Athena M. Cherry, Sibel Kantarci, Hutton Kearney, Ankita Patel, Gordana Raca, Deborah I. Ritter, Sarah T. South, Erik C. Thorland, Daniel Pineda-Alvarez, Swaroop Aradhya and Christa Lese Martin

Genetics in Medicine (2021) 23:2230; https://doi.org/10.1038/s41436-021-01150-9

Correction to: Genetics in Medicine (2020) 22:245-257; https://doi. org/10.1038/s41436-019-0686-8, published online 06 November 2019
The original version of this article unfortunately contained a mistake. In Table 1, the default score for item $5 \mathrm{H}$ should be 0.15 instead of 0.30 . The corrected section 5 from Table 1 is given below.

\section{Section 5: Evaluation of inheritance pattern/family history for patient being studied}

Observed copynumber loss is de novo

Observed copynumber loss is inherited

Observed copynumber lossnonsegregations Other
5A. Use appropriate category from de novo scoring section in section 4.

5B. Patient with specific, well-defined phenotype and no family history. CNV is inherited from an apparently unaffected parent.

5C. Patient with nonspecific phenotype and no family history. CNV is inherited from an apparently unaffected parent.

5D. CNV segregates with a consistent phenotype observed in the patient's family.

5E. Use appropriate category from nonsegregation section in section 4.

5F. Inheritance information is unavailable or uninformative.

5G. Inheritance information is unavailable or uninformative. The patient phenotype is nonspecific, but is consistent with what has been described in similar cases.

$\mathbf{5 H}$. Inheritance information is unavailable or uninformative. The patient phenotype is highly specific and consistent with what has been described in similar cases.
Use de novo scoring categories from section $4(4 A-4 D)$ to determine score

-0.30 (range: 0 to -0.45 )

-0.15 (range: 0 to -0.30 )

Use segregation scoring categories from section $4(4 F-4 H)$ to determine score

Use nonsegregation scoring categories

$-0.45$ from section 4 (4I-4K) to determine score

0

0.10 (range: 0 to 0.15 )

0.15 (range: 0 to 0.30 ) 